\title{
Seroprevalence and Risk Factors of Fascioliasis in Yaks, Bos grunniens, from Three Counties of Gansu Province, China
}

\author{
Xiao-Xuan Zhang ${ }^{1,2}$, Sheng-Yong Feng'2, Jian-Gang Ma, , Wen-Bin Zheng ${ }^{1,2}$, Ming-Yang Yin², Si-Yuan Qin², \\ Dong-Hui Zhou', Quan Zhao,*, Xing-Quan Zhu',** \\ ${ }^{1}$ College of Animal Science and Technology, Jilin Agricultural University, Changchun, Jilin 130118, China; ${ }^{2}$ State Key Laboratory of Veterinary \\ Etiological Biology, Key Laboratory of Veterinary Parasitology of Gansu, Lanzhou Veterinary Research Institute, Chinese Academy of Agricultural \\ Sciences, Lanzhou, Gansu 730046, China
}

\begin{abstract}
The aim of this study was to determine the seroprevalence and risk factors of fascioliasis in yaks, Bos grunniens, from 3 counties of Gansu Province in China. A total of 1,584 serum samples, including 974 samples from white yaks from Tianzhu, 464 from black yaks from Maqu, and 146 from black yaks from Luqu County, were collected and analyzed using ELISA to detect IgG antibodies against Fasciola hepatica. The overall $F$. hepatica seroprevalence was $28.7 \%$ $(454 / 1,584)$, with $29.2 \%$ in white yaks $(284 / 974)$ and $27.9 \%$ in black yaks (170/610). The seroprevalence of $F$. hepatica in yaks from Tianzhu, Luqu, and Maqu was $29.2 \%, 22.6 \%$, and $29.5 \%$, respectively. Female yaks (30.9\%) had higher F. hepatica seroprevalence than male yaks (23.4\%). Also, F. hepatica seroprevalence varied by different age group from $24.1 \%$ to $33.8 \%$. Further, the seroprevalence ranged from $21.8 \%$ to $39.1 \%$ over different seasons. Interestingly, the season and age of yaks were associated with $F$. hepatica infection in yaks in the investigated areas. These findings provided a basis for further studies on this disease in yaks from 3 counties of Gansu Province in northwestern China, which may ultimately support the development of effective control strategies of fascioliasis in these areas.
\end{abstract}

Key words: Fasciola hepatica, Bos grunniens, yak, seroprevalence, ELISA, China

The white yak is a unique yak breed and precious semi-wild animal in China [1]. Approximately 50,000 white yaks live only in Tianzhu Tibetan Autonomous County (TTAC), Gansu Province, northwestern China, where low air pressure, low temperature, and low oxygen content exist [1]. Milk and meat of yaks (especially white yaks) is the key source of income for local Tibetans in TTAC (with an altitude of 2,050-4,874 m above the sea level), Maqu (3,500-3,800 $\mathrm{m}$ above the sea level), and Luqu (2,900-4,287 $\mathrm{m}$ above the sea level), Gansu Province, China [1]. Furthermore, most of Tibetans and their livestock (white yaks and black yaks) live in mountains in Gansu, and due to the lack of infrastructure, Tibetans and yaks drink the water from the river nearby.

Fasciola hepatica and Fasciola gigantica are the major causative agents of fascioliasis, an economically important disease in

- Received 3 March 2016, revised 12 November 2016, accepted 24 December 2016. *Corresponding author (zhaoquan0825@163.com; xingquanzhu1@hotmail.com) (c) 2017, Korean Society for Parasitology and Tropical Medicine

This is an Open Access article distributed under the terms of the Creative Commons Attribution Non-Commercial License (http://creativecommons.org/licenses/by-nc/4.0) which permits unrestricted non-commercial use, distribution, and reproduction in any medium, provided the original work is properly cited. livestock $[2,3]$. F. hepatica infection usually occurs in temperate zones [4], and F. gigantica occurs in tropical and subtropical areas. Fasciola infection is caused by ingestion of encysted metacercariae through oral route, and the affected host exhibits symptoms of liver damage, loss of productivity, and even death [5].

Recently, fascioliasis is of increasing concern because Fasciola not only has a worldwide distribution, but also threatens human health. For example, up to 2.4 million humans are infected and about 180 million are at risk worldwide [6]. Fascioliasis may also cause great economic losses as indicated by the loss of more than US $\$ 3,000$ million and more than 600 million animals infected per year worldwide [7]. In view of this impact, large numbers of investigations concerning the prevalence of Fasciola in bovines have been conducted in the world $[5,8]$.

In China, information about the prevalence of Fasciola in yaks (Bos grunniens) is limited, a few previous studies demonstrated that $F$. hepatica is the predominant Fasciola species in yaks, and these were published in Chinese journals $[9,10]$. The objective of the present study was to investigate the seropreva- 
Table 1. Seroprevalence of Fasciola hepatica infection in yaks (Bos grunniens) in Gansu province, northwestern China by ELISA

\begin{tabular}{llccccc}
\hline Variable & \multicolumn{1}{c}{ Category } & No. tested & No. positive & $\%(95 \%$ Cl) & $P$-value & OR (95\% Cl) \\
\hline Region & Luqu & 146 & 33 & $22.6(15.82-29.39)$ & 0.23 & Reference \\
& Tianzhu & 974 & 284 & $29.2(26.30-32.01)$ & $1.41(0.93-2.13)$ \\
& Maqu & 464 & 137 & $29.6(25.38-33.68)$ & $1.44(0.93-2.22)$ \\
Gender & Male & 471 & 110 & $23.4(19.53-27.18)$ & 0.2 & Reference \\
& Female & 1,113 & 344 & $30.9(28.19-33.62)$ & $1.47(1.15-1.88)$ \\
Species & Black yaks & 610 & 170 & $27.9(24.31-31.43)$ & 0.58 & Reference \\
& White yaks & 974 & 284 & $29.2(26.30-32.01)$ & $1.07(0.85-1.33)$ \\
Age & O<year $\leq 1$ & 286 & 69 & $24.1(19.17-29.09)$ & 0.02 & Reference \\
& 1<years $\leq 2$ & 292 & 81 & $27.7(22.60-32.88)$ & $1.21(0.83-1.75)$ \\
& 2<years $\leq 4$ & 521 & 140 & $26.9(23.07-30.68)$ & $1.16(0.83-1.61)$ \\
Season & 4<years & 485 & 164 & $33.8(29.60-38.03)$ & $1.61(1.16-2.24)$ \\
& Summer (Apr. to Jun.) & 354 & 77 & $21.8(17.45-26.05)$ & $<0.0001$ & Reference \\
& Spring (Jan. to Mar.) & 428 & 94 & $22.0(18.04-25.89)$ & $1.01(0.72-1.42)$ \\
Total & Autumn (Jul. to Sep.) & 467 & 152 & $32.6(28.30-36.80)$ & $1.74(1.26-2.39)$ \\
\hline
\end{tabular}

lence of $F$. hepatica infection in white yaks and black yaks in Gansu Province, northwestern China, with the goal to provide "baseline" information for estimating the effectiveness of future control strategies against $F$. hepatica infection in yaks in China.

The study was approved by the Animal Ethics Committee of Lanzhou Veterinary Research Institute, Chinese Academy of Agricultural Sciences (approval no. LVRIAEC2013-010). A total of 1,584 serum samples were randomly collected from 3 counties (974 from Tianzhu, $36^{\circ} 31^{\prime}-37^{\circ} 55^{\prime} \mathrm{N}, 102^{\circ} 07^{\prime}$ $103^{\circ} 46^{\prime} \mathrm{E} ; 464$ from Maqu, $33^{\circ} 06^{\prime}-34^{\circ} 30^{\prime} \mathrm{N}, 100^{\circ} 45^{\prime}-102^{\circ} 29^{\prime}$ E; 146 from Luqu County, $33^{\circ} 58^{\prime}-34^{\circ} 48^{\prime} \mathrm{N}, 101^{\circ} 35^{\prime}-102^{\circ} 58^{\prime}$ E) between April 2013 and March 2014. The 4 seasons from spring to winter were defined as January-March, April-June, July-September, and October-December, respectively. All the investigation sites have a short period of warm season (June to October). The blood samples were transported quickly on ice to the laboratory, and centrifuged at 3,000 $\mathrm{g}$ for $10 \mathrm{~min}$, and then serum was obtained, and stored at $-20^{\circ} \mathrm{C}$ until used. All information of the yaks examined, i.e., body color, age and sex, geographical region, and surveyed season, were recorded.

The level of antibodies against $F$. hepatica were examined using ELISA by the SVANOVIR ${ }^{\circledR}$ F. hepatica-Ab ELISA test (Svanova, Boeringer, Uppsala, Sweden) according to the manufacturer's recommendations. The excretory-secretory (ES)-antigen did not cross-react with Ostertagia ostertagi infections. The ELISA kit used in this study has a high specificity $(88 \%)$ and sensitivity $(92 \%)$, and has been widely used for detection of $F$. he- patica infection in bovine previously [11,12]. The cut-off was set at 0.3 , meaning that ratios below this level were considered as negative. The positive and negative controls were supplied in the kit and included in each test.

The variation in $F$. hepatica prevalence $(\gamma)$ of yaks from different geographical locations $(\chi 1)$, species $(\chi 2)$, genders $(\chi 3)$, season groups $(\chi 4)$, and ages $(\chi 5)$ was analyzed by the chisquare test using SAS version 9.1 (SAS Institute Inc., Cary, North Carolina, USA) [13]. Each of these variables was included in the binary Logit model as an independent variable based on the multivariable regression analysis. The best model was judged by Fisher's scoring algorithm. All tests were 2-sided, and values of $P<0.05$ were considered statistically significant. Odds ratios (ORs) and their 95\% confidence intervals (95\% CIs) were estimated to explore the strength of the association between $F$. hepatica-seropositivity and the conditions investigated.

Of the 1,584 tested serum samples, 454 (28.7\%) were positive for F. hepatica antibody, with $29.2 \%$ (284/974) in white yaks and $27.9 \%(170 / 610)$ in black yaks. The positive rate in female yaks $(30.9 \%, 344 / 1,113)$ was higher than in males $(23.4 \%, 110 / 471)$ (Table 1). The seroprevalence was $29.2 \%$ (284/974), 22.6\% (33/146), and 29.5\% (137/464) in yaks from Tianzhu, Luqu, and Maqu County, in Gansu Province, China, respectively. They were different by age groups and ranged from $24.1 \%(69 / 286)$ to $33.8 \%(164 / 485)$. The seasonal prevalences were $22.0 \%(94 / 428), 21.8 \%(77 / 354)$, $32.6 \%$ (152/467), and 39.1\% (131/335) in spring, summer, 
autumn, and winter, respectively (Table 1).

In the present study, the overall $F$. hepatica seroprevalence was $28.7 \%(454 / 1,584)$, which was lower than that reported in yaks in Xinghai tested by autopsy method $[9,10]$, cattle in Switzerland [14], Poland [14], Australia [15], Ireland [16], and Vietnam [17] examined by ELISA, but higher than that of cattle in Spain [5] and Denmark [18], cow in Germany [19] tested by ELISA. It has been demonstrated that $F$. hepatica infection mainly occurs in cattle [20] and goats [20], otherwise, F. gigantica usually infect water buffaloes. So, these differences could be related to the animal susceptibility to fascioliasis. Moreover, local climatic conditions, farming regimen, animal welfare, as well as sampling time and sample sizes may also affect the results.

Table 1 presents the relationship between $F$. hepatica-positivity in yaks based on the univariate analysis. The impacts of multiple variables on $F$. hepatica were evaluated by forward stepwise logistic regression analysis using Fisher's scoring technique. In the final model, 2 variables had effects on the disease, described by the equation " $y=-0.3731 \times 4-0.2329 \times 5+$ 2.5191". Season had a strong effect on the risk of $F$. hepatica, which was in accordance with previous studies [10,21-24].

Almost all life in the investigated areas (Maqu, Luqu, and Tianzhu counties) drink water from the river nearby. The infected yaks can shed eggs into the environment (including rivers), which is a potential source for snail (can survive during July to October) infection, and the infected snails can shed cercariae into the environment. Cercariae then develop into metacercariae (in rivers and/or grass) which infect the definitive hosts (including yaks). This forms the vicious circle. Statistical analysis suggested that the $F$. hepatica seroprevalence was significantly higher in yaks in winter $(39.1 \%, 95 \%$ CI 33.9$44.3)$ and autumn (32.6\%, 95\% CI 28.3-36.8) than yaks in spring (22.0\%, 95\% CI 18.0-25.9) and summer (21.8\%, 95\% CI 17.5-26.1, $P<0.0001$ ) (Table 1 ). This may be due to the fact that the climatic conditions in the surveyed regions during July to October were favorable for the development of $F$. hepatica. These results also suggest that temperature should be considered when carrying out control programs in the investigation areas.

Mammalian hosts acquire F. hepatica infection through ingestion of contaminated vegetation or water containing viable metacercariae of $F$. hepatica throughout their lifetimes [25,26], so older hosts have more opportunities to ingest encysted dormant larvae. In the present study, age was considered as a risk factor associated with $F$. hepatica infection in yaks.

Multivariable regression analysis showed that yaks of more than 4 years $(\mathrm{OR}=1.61,95 \% \mathrm{CI} 1.16-2.24)$, of 2-4 years $(\mathrm{OR}=1.16,95 \% \mathrm{CI} 0.83-1.61)$ and of $1-2$ years $(\mathrm{OR}=1.21$, $95 \%$ Cl $0.83-1.75$ ) has a 1.61 times, 1.16 times, and 1.21 fold higher risk of infection compared to yaks of 0-1 year (24.1\%), respectively (Table 1). The trend of $F$. hepatica seroprevalence increased with age of yaks, which was in accordance with previous reports of an age-cumulative effect in the seroprevalence of $F$. hepatica in cattle $[25,27]$.

In the present study, a higher seroprevalence was found in Maqu County of higher altitude than that in Luqu and TTAC with lower altitudes, although the difference was not statistically significant $(P=0.23)$. This could be related to many factors, such as farming regimen, animal welfare, as well as sampling time and sample sizes, so whether there is a direct relationship between altitude and fascioliasis prevalence should be determined in further studies. A previous study indicated that F. gigantica also infects yaks in India [28]. In China, F. gigantica infection has also been reported in humans [29] and water buffaloes [30], but no information concerning F. giganti$c a$ infection in yaks was recorded. Therefore, whether F. gigantica can infect yaks in China should be investigated in future studies.

Taken together, the present survey indicated high $F$. hepatica seroprevalence $(28.7 \%, 454 / 1,584)$ in yaks from 3 counties of Gansu Province in northwestern China, with 29.2\% (284/974) and $27.9 \%$ (170/610) in white yaks and black yaks, respectively, which can cause economic losses to the local yak industry, and also has a potential threat to the health of Tibetans in these areas. Moreover, season and age of yak were found to be correlated with $F$. hepatica infection in yaks in the investigated areas. This information provides new local disease surveillance data, which can be very valuable for any future control interventions.

\section{ACKNOWLEDGMENTS}

Project support was provided by the Special Fund for Agroscientific Research in the Public Interest (grant no. 201303037), the National Key Basic Research Program (973 Program) of China (grant no. 2015CB150300), and the Key Scientific and Technological Project of Jilin Province, China (grant no. 2014 0204068NY). We thank Dr. Hany M. Elsheikha, Associate Professor of Parasitology at the Faculty of Medicine and Health 
Sciences, School of Veterinary Medicine and Science, the University of Nottingham, UK, for useful comments and suggestions, which significantly improved the clarity of this manuscript.

\section{CONFLICT OF INTEREST}

We have no conflict of interest related to this work.

\section{REFERENCES}

1. Ma C. Study on sustainable development strategies of Tianzhu white yak industry. J Domest Anim Ecol 2008; 29: 133-135.

2. Andrews SJ. The life cycle of $F$. hepatica. In Dalton JP, ed, Fasciolosis. Wallingford, Oxon, UK. CABI Publishing. 1998, pp 1-20.

3. Sunita K, Habib M, Kumar P, Singh VK, Husain SA, Singh DK. Inhibition of acetylcholinesterase and cytochrome oxidase activity in Fasciola gigantica cercaria by phytoconstituents. Acta Trop 2016; 154: 19-24.

4. Charlier J, Vercruysse J, Morgan E, van Dijk J, Williams DJ. Recent advances in the diagnosis, impact on production and prediction of Fasciola hepatica in cattle. Parasitology 2014; 141: 326335 .

5. Arias MS, Piñeiro P, Sánchez-Andrade R, Suárez JL, Hillyer GV, Díez-Baños P, Paz-Silva A, Morrondo P. Relationship between exposure to Fasciola hepatica in roe deer (Capreolus capreolus) and cattle extensively reared in an endemic area. Res Vet Sci 2013; 95: 1031-1035.

6. Chen IX, Chen MX, Ai L, Xu XN, Jiao JM, Zhu TJ, Su HY, Zang W, Luo JJ, Guo YH, Lv S, Zhou XN. An outbreak of human fascioliasis gigantica in Southwest China. PLoS One 2013; 8: e71520.

7. Piedrafita D, Spithill TW, Smith RE, Raadsma HW. Improving animal and human health through understanding liver fluke immunology. Parasite Immunol 2010; 32: 572-581.

8. Elelu N, Ambali A, Coles GC, Eisler MC. Cross-sectional study of Fasciola gigantica and other trematode infections of cattle in Edu Local Government Area, Kwara State, north-central Nigeria. Parasit Vectors 2016; 9: 470.

9. Chai ZM. Prevalence of Fasciola hepatica infection in yaks in Xinghai, Qinghai. Chinese J Vet Med 2012; 12: 43-44.

10. Chai ZM. Prevalence of Fasciola hepatica infection in yaks in Xinghai. Anim Hu Vet Med 2013; 2: 112.

11. Charlier J, De Cat A, Forbes A, Vercruysse J. Measurement of antibodies to gastrointestinal nematodes and liver fluke in meat juice of beef cattle and associations with carcass parameters. Vet Parasitol 2009; 166: 235-240.

12. Charlier J, Hostens M, Jacobs, J, van Ranst B, Duchateau L, Vercruysse J. Integrating fasciolosis control in the dry cow management: the effect of closantel treatment on milk production. PLoS One 2012; 7: e43216.

13. Zhang XX, Jiang J, Cai YN, Wang CF, Xu P, Yang GL, Zhao Q.
Molecular characterization of Enterocytozoon bieneusi in domestic rabbits (Oryctolagus cuniculus) in Northeastern China. Korean J Parasitol 2016; 54: 81-85.

14. Karanikola SN, Krücken J, Ramünke S, de Waal T, Höglund J, Charlier J, Weber C, Müller E, Kowalczyk SJ, Kaba J, von Samson-Himmelstjerna G, Demeler J. Development of a multiplex fluorescence immunological assay for the simultaneous detection of antibodies against Cooperia oncophora, Dictyocaulus viviparus and Fasciola hepatica in cattle. Parasit Vectors 2015; 8: 335.

15. Elliott TP, Kelley JM, Rawlin G, Spithill TW. High prevalence of fasciolosis and evaluation of drug efficacy against Fasciola hepati$c a$ in dairy cattle in the Maffra and Bairnsdale districts of Gippsland, Victoria, Australia. Vet Parasitol 2015; 209: 117-124.

16. Selemetas N, Phelan P, O'Kiely P, de Waal T. The effects of farm management practices on liver fluke prevalence and the current internal parasite control measures employed on Irish dairy farms. Vet Parasitol 2015; 207: 228-240.

17. Nguyen TG, Le TH, Dao TH, Tran TL, Praet N, Speybroeck N, Vercruysse J, Dorny P. Bovine fasciolosis in the human fasciolosis hyperendemic Binh Dinh province in Central Vietnam. Acta Trop 2011; 117: 19-22.

18. Olsen A, Frankena K, Bødker R, Toft N, Thamsborg SM, Enemark HL, Halasa T. Prevalence, risk factors and spatial analysis of liver fluke infections in Danish cattle herds. Parasit Vectors 2015; 8: 160.

19. Kuerpick B, Conraths FJ, Staubach C, Fröhlich A, Schnieder T, Strube C. Seroprevalence and GIS-supported risk factor analysis of Fasciola hepatica infections in dairy herds in Germany. Parasitology 2013; 140: 1051-1060.

20. Yuan W, Liu JM, Lu K, Li H, Duan MM, Feng JT, Hong Y, Liu YP, Zhou Y, Tong LB, Lu J, Zhu CG, Jin YM, Cheng GF, Lin JJ. Molecular identification and seasonal infections of species of Fasciola in ruminants from two provinces in China. J Helminthol 2016; 90: 359-363.

21. Rognlie MC, Dimke KL, Potts RS, Knapp SE. Seasonal transmission of Fasciola hepatica in Montana, USA, with detection of infected intermediate hosts using a DNA-based assay. Vet Parasitol 1996; 65: 297-305.

22. Cruz-Mendoza I, Ibarra-Velarde F, Quintero-Martínez MT, Naranjo-García E, Lecumberri-López J, Correa D. Seasonal transmission of Fasciola hepatica in cattle and Lymnaea (Fossaria) humilis snails in central Mexico. Parasitol Res 2005; 95: 283-286.

23. Waller PJ. Sustainable nematode parasite control strategies for ruminant livestock by grazing management and biological control. Anim Feed Sci Technol 2006; 126: 277-289.

24. Valero MA, Perez-Crespo I, Khoubbane M, Artigas P, Panova M, Ortiz P, Maco V, Espinoza JR, Mas-Coma S. Fasciola hepatica phenotypic characterization in Andean human endemic areas: valley versus altiplanic patterns analysed in liver flukes from sheep from Cajamarca and Mantaro, Peru. Infect Genet Evol 2012; 12: 403-410.

25. Robinson MW, Dalton JP, O'Brien BA, Donnelly S. Fasciola hepatica: the therapeutic potential of a worm secretome. Int $J$ Parasitol 
2013; 43: 283-291.

26. Kang BK, Jung BK, Lee YS, Hwang IK, Lim H, Cho J, Hwang JH, Chai JY. A case of Fasciola hepatica infection mimicking cholangiocarcinoma and ITS-1 sequencing of the worm. Korean J Parasitol 2014; 52: 193-196.

27. Sánchez-Andrade R, Paz-Silva A, Suárez JL, Panadero R, Pedreira J, López C, Díez-Baños P, Morrondo P. Influence of age and breed on natural bovine fasciolosis in an endemic area (Galicia, NW Spain). Vet Res Commun 2002; 26: 361-370.
28. Kuchai JA, Chishti MZ, Ahmad F, Rasool M. Prevalence of trematode parasitic infestation in Yak of Ladakh (Bos grunniens). Pakistan J Wildl 2010; 1: 64-66.

29. Chen JX, Chen MX, Ai L, Xu XN, Jiao JM, Zhu TJ, Su HY, Zang W, Luo JJ, Guo YH, Lv S, Zhou XN. An outbreak of human fascioliasis gigantica in Southwest China. PLoS One 2013; 8: e71520.

30. Huang WY, He B, Wang CR, Zhu XQ. Characterisation of Fasciola species from Mainland China by ITS-2 ribosomal DNA sequence. Vet Parasitol 2004; 120: 75-83. 